\title{
Smart Grid Measurement Uncertainty: Definitional and Influence Quantity Considerations
}

\author{
William Dickerson ${ }^{1}$, Allen Goldstein ${ }^{2}$, Harold Kirkham ${ }^{3}$, Kenneth Martin ${ }^{4}$, \\ Andrew Roscoe ${ }^{5}$, Roel de Vries ${ }^{6}$, Paul Wright ${ }^{7}$ \\ ${ }^{1}$ Arbiter Systems, Paso Robles, CA, USA, bdickerson@arbiter.com \\ ${ }^{2}$ National Institute for Standards and Techology, NIST, Gaitherburg, MD, USA, allen.goldstein@ nist.gov \\ ${ }^{3}$ Pacific Northwest National Lab, PNNL, Richland, WA, USA \\ ${ }^{4}$ EPG, Pasadena, CA, USA, martin@electricpowergroup.com \\ ${ }^{5}$ University of Strathclyde, Glasgow, Scotland, andrew.j.roscoe@strath.ac.uk \\ ${ }^{6}$ Arbiter Systems, Almelo, The Netherlands, roel.devries@arbitersystems.eu \\ ${ }^{7}$ National Physical Laboratory, NPL, Teddington, UK, paul.wright@npl.co.uk
}

\begin{abstract}
Measurement uncertainty, in general terms, is an expression of the quality of a measurement. It is typically quantified by defining the location and spread of the distribution of a hypothetical infinitely large number of measurements of the thing being measured. The Guide to the Expression of Uncertainty in Measurement (GUM) classifies uncertainties according to whether they are evaluated by statistical means or not. The GUM also mentions that an incomplete definition of the measurand can give rise to large uncertainty in the result. For some of the quantities measured in the Smart Grid, it may be that this "definitional uncertainty" could be a major source of problems. Influence quantities may have a bearing on the result of a measurement without being the subject of the measurement. For example, signal harmonics, noise and temperature effects, while not desired as part of a measurement, exist in power system signals being measured, and they have (sometimes significant) effects on the measurement process.
\end{abstract}

Index Terms - measurement, smart grid, uncertainty.

\section{INTRODUCTION}

Metrologists are well aware of measurement uncertainty, and methods of dealing with it and expressing it are described in such documents as the 'Guide to the expression of uncertainty in measurement' (GUM)[1]. The GUM deals comprehensively with traditional sorts of measurement uncertainty those related to factors such as uncertainty of the reference artifact(s) used in a measurement, drift, temperature sensitivity, nonlinearity, and noise. Metrologists are experienced in dealing with these forms of uncertainty.

However, in real world applications such as the Smart Grid, additional forms of uncertainty arise. This paper addresses two such forms: (1) definitional uncertainty; and (2) uncertainty due to influence quantities.

\section{A. Definitional uncertainty}

Definitional uncertainty arises from the question: "What exactly is it we are trying to measure?" For instance, clause 1.2 of the GUM says: "This Guide is primarily concerned with the expression of uncertainty in the measurement of a well-defined physical quantity - the measurand - that can be characterized by an essentially unique value." (The italics are ours.) In the Smart Grid, what makes measurements useful is that the values are not fixed, but varying. To control, protect and meter the grid we monitor dynamically varying quantities such as current. There is little guidance respecting how to define the relationship between time and the measurand(s), and what result is expected (or desired) when a physical quantity varies in time.

Further, some quantities (such as frequency or rms voltage) are not directly physically realizable. Surely we want to measure these things as well; but what should be the definition of such things, particularly when they vary with time, and measurands include allocated timestamps with microsecond resolution? At the present time, different users apply different interpretations of the measurand definitions; as the GUM states in 3.3.2 $\mathrm{a}$ and $\mathrm{b}$, incomplete definition or imperfect realization of the measurand, which we call definitional uncertainty, is a significant source of uncertainty.

\section{B. Representationalism vs Operationalism}

Measurement methods have a number of ways of dealing with such problems. They are all concerned with using some physical aspect of the the real world to find a conceptual value that allows us to understand something about the real world. One might imagine these methods spread along a continuum. At one end of the continuum are the representationalist methods. The quantity to be measured is represented by an equation. It is surely this kind of measurement that Kelvin was thinking of when he famously wrote

\footnotetext{
... when you can measure what you are speaking about, and express it in numbers, you know something about it; but when you cannot measure it, when you cannot express it in numbers, your knowledge is of a meager and unsatisfactory kind: it may be the beginning of knowledge, but you have scarcely, in your thoughts advanced to the stage of science... [2]
}

At the other end of the continuum of measurements are the operationalist methods in which the result depends not on the definition or the mathematics applied to the information available, but on the method used. Operationalism is exemplified 
by the Rockwell hardness tester, which makes a dent in a test object, and by the measurement of intelligence, something that can still be measured even without a firm definition.

An obvious problem with operationalist methods, as far as power engineering is concerned, is that it is not generally clear exactly what is being measured. Most electrical engineers have a natural bias toward representationalism. But in reality, few measurements can be regarded as "pure" in terms of being at one the end of the continuum. And we should bear in mind that the application of filters to signals moves the process of measurement toward the operationalist end. In fact, the addition of filtering to a signal, by making it less obvious exactly what is being measured, may increase definitional uncertainty, because of the potential mismatch between what is actually contributing to the result of a measurement and what is supposed by the observer.

\section{Influence quantity uncertainties}

In the Smart Grid, we must make most of our measurements in the presence of interfering or influence quantities, which we might refer to as pollution. These are things (e.g., harmonics, interharmonics, noise, phase jumps, and phase imbalance) which are almost always present in the real grid at some level, often at the same time, and which are themselves time varying.

These things are not part of the signal that we normally expect to be measuring, and they often complicate extracting the wanted "essentially unique value." Essentially, the pollution creates an added definitional uncertainty: since these quantities are not included in the definition, they add to the definitional uncertainty.

Even if we do include these quantities in the definition, and require them not to be included in the measurement, practical implementation issues limit the extent to which they can be excluded. Filters, for instance, can only approximate a 'brick wall' transfer function; realizable filters always include a transition band, in which undesired pollution is attenuated, but not completely removed.

\section{Beyond the GUM}

In making our measurements, we are going beyond guidance provided in the GUM, which assumes that definitional uncertainty is negligible compared to other sources of uncertainty. The GUM does not itself use the term "definitional uncertainty," but the point is made in the Introduction of the VIM, the International Vocabulary of Measurement, which observes (page ix) that "In the GUM, the definitional uncertainty is considered to be negligible with respect to the other components of measurement uncertainty.'[3]

In this paper, we have given much of our attention to the problem of frequency measurement. There are two reasons: first, several of us have already done significant work in this area so we are familiar with it; and second, because the frequency measurement is one where we have found users very likely to notice the effects of small, unexpected errors.

\section{Definitional UnCERTAinty}

The real quantity to be measured is described by a definition that some call the measurand (though that word is also used for the realized quantity by some metrologists). The result of the measurement is (ideally) a value of the measurand, a model of the realized quantity. While we acknowledge that the realized quantity might be affected by influence quantities such as signal distortion, we sometimes overlook the fact that the definition itself might not be perfect.

GUM offers the view that a quantity cannot be completely described without an infinite amount of information. An incomplete definition (and GUM implies that all practical definitions must be incomplete) thus implies an associated uncertainty.

For example, consider a measurement of single-phase AC frequency, while frequency varies with time in an arbitrary manner. To begin with, we observe that while the word frequency is linguistically associated with some sort of repetitive action or signal, mathematically it applies to something that is constant over all time: a sinusoid, for example. The domain of the sine function is between plus and minus one in amplitude and plus and minus infinity in time. Therefore, we should be cautious about applying the conventional interpretations to both our words and our work. A representationalist approach to the definition we call the measurand would be

- a mathematical equation involving a non-constant "frequency" which applies over some particular time.

An operationalist approach to the problem would involve

- a definition of window/filter lengths/shapes and/or assessment methods

In the representationalist method, frequency is simply the coefficient of a term in the equation, whereas the operationalist method derives its result from a more complicated method. We expect that, for a given input signal, the two schemes will produce the same result. Indeed, a method of confirming proper operation is required in either case. Thus, some sort of definition of what is meant by the word "frequency" must be found. It is far from simple.

To illustrate the problem, one has only to compare the definitions of frequency within, for example, IEC 61000-430[4] and IEEE Std C37.118.1[5], which are totally different. Attempts to define the meaning of a single measurand "frequency," within a three-phase system that is potentially faulted, unbalanced, or connected in negative sequence, lead to even more ambiguity, conflict and confusion, given the present state of the art.

Similar problems present for other measurands. For instance, if we are to estimate an rms voltage or current for a time-varying signal, should that estimate include energy from harmonics, interharmonics and/or low-frequency transients arising in instrument transformers? Note also that the measurement of an rms value requires either the specification of an averaging interval and window-shaping method, or knowledge of the period (that is, the time derived from a knowledge of the frequency) over which the measurement is 
to be made. There is no right and wrong in this: while the GUM deprecates the use of the term "true value," it is shown in [6] that the idea of there existing a true value of a measured quantity does not apply to this kind of measurement.

Without a clearly stated and well-understood definition (the measurand) of the object of our measurement process (the realized quantity), we cannot communicate the meaning of the result of the measurement process to the user. A purpose of this paper is to stimulate discussion of the topic of the proper definitions for the measurement of frequency, and of the measurands used in the Smart Grid in general.

The proper refinement of the definitions holds out the possibility that the measurement results will become more useful for the intended applications. We are not attempting to provide all the answers in this paper; rather, we are only beginning to scratch the surface. Bearing in mind the GUM's pessimistic warning that a perfect measurand requires an infinite amount of information, we hope to move the community toward the goal of a better definition or definitions, while keeping them as concise as the requirements allow.

\section{INFLUENCE QUANTITY UNCERTAINTY}

Suppose for our earlier frequency-estimation example that there may be interharmonics present. Presumably we wish to know the value of the frequency of the $\approx 50 \mathrm{~Hz}$ fundamental of the power signal. One common method of measurement, zero crossings, is quite accurate for an unimpaired signal, but the presence of non-harmonically-related signal content degrades accuracy badly [7].

Other estimation methods have varying sensitivity to such influence quantities. For instance, a Fourier transform approach can separate the signals into different 'bins,' and the phase of the desired signal can be used to calculate frequency as the rate of change of phase angle between two successive transforms. With suitable windowing/filtering, it is possible to provide a significant amount of reduction of the interfering interharmonics. But short windows of one or two cycles provide only limited reduction of nearby interharmonics.

All practical measurement methods necessarily involve a time window of non-zero duration. "Instantaneous" measurements imply reducing the window length to zero or near-zero, and for the case of frequency estimation, such methods are the subject of much discussion [8][9][10].

Frequency is a term associated with repetitive action, and if the duration of the signal is restricted to less than one cycle, the manner of repetition must be assumed by the analyst. In a Fourier analysis, for example, the mathematics actually are based on an infinitely-repeating signal, and for a short stretch of signal, corrections must usually be made for what is called "spectral leakage," an error that occurs when the window duration is not an exact multiple of the signal period. This problem is not new; it was first described in 1848 by Wilbraham [11].

A review paper [12][13] points out that if the spectrum of an amplitude change and a frequency change overlap, their interpretation is problematical. Cohen [14] reviews 213 studies of the effect of changing frequency and time-limited windows, and observes that there have been hundreds of plausible methods of representing the signal, none of which seem to be quite right.

Excessive reduction of window length has severe consequences for influence quantity uncertainty. AC measurements of less than one cycle duration are extremely vulnerable to many influence quantities, including harmonics. For windows of at least one cycle length, harmonic and unbalance influences can be dealt with relatively well. However, handling more problematic influences like noise and interharmonics usually requires longer windows.

Given that the window may therefore last several (perhaps tens of) cycles of the quantity of interest, it becomes increasingly likely that the signal itself may contain an influence quantity as gross as an AC phase or frequency step. The step can occur anywhere in the window, from the very start to the very end. While we can create both a mathematical equation and a physically-realized signal that embody a step in phase or frequency, we are not yet aware of any ways to practically and reliably identify and separate such gross influence quantities from the underlying measurand, in real time. When the step in influence quantity occurs at the very end of the window, i.e. the most recent samples, the practical challenge is largest. The measurement system can have no knowledge of what is coming next. How do we reflect these problems in definitions, requirements and standards?

The phase-step phenomenon, in the context of frequency measurement, is also an excellent example of the definitional uncertainty problem. A phase step may be considered to be a part of the frequency measurand, or it may be considered to be an influence quantity, depending on your point of view, the eventual application, and the signal model being employed [15]).

\section{FURTHER EXAMPLES}

\section{A. Smart Grid modal analysis}

Consider, for example, system stability analysis. One of the tools often used for this is Fourier analysis of a series of frequency measurements. This gives us a plot such as Fig. 1, in which periodic frequency excursions due to system resonant modes can be seen as peaks in a mode (sideband) frequency vs. time graph.

These graphs are often shown in 'waterfall' form, allowing the operator to visualize the variation in magnitude of these peaks with time. Unstable modes, i.e. underdamped modes, grow in magnitude over time, and left unchecked can cause serious problems up to and including cascading failure of the grid.

Note, however, the magnitude of these peaks. They often represent a variation in the system frequency - a frequency modulation - of only a few milliHertz magnitude. Indeed the 'peaks' in Fig. 1 at $\approx 8 \mathrm{~Hz}$ are less than that, around $0.3 \mathrm{mHz}$ in 
magnitude. Communications theory tells us that this represents a corresponding phase modulation as shown by:

$$
\Delta \Phi=\Delta F / F_{m}
$$

where $F_{m}$ is the modulation rate (sideband frequency).

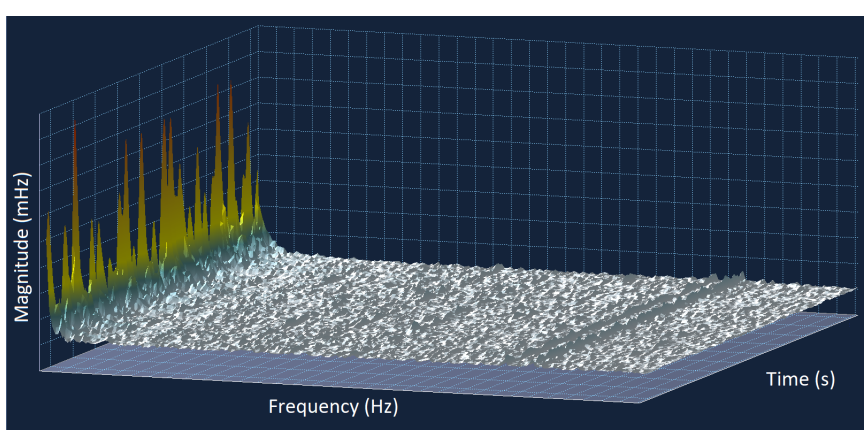

Fig. 1: Sideband magnitude vs. time and frequency

The phase modulation is thus also often only a few milliradians, and can be less; in this case it is $\approx 0.04 \mathrm{mrad}$. From this we can see the power of this tool: very small deviations in phase angle caused by system modes can easily be seen. But, the requirements in the standards for PMU performance are larger than the effects we would like to examine. One percent TVE corresponds to 10 milliradians of phase error which can be far greater than the phase deviation caused by the system mode. Even the frequency requirements are often larger than the signal to be visualized: $5 \mathrm{mHz}$ in steady state, and larger errors are allowed in the presence of harmonics and out-of-band interfering signals [5][16].

Clearly in this case, our existing definition of the measurand (in [5] and [16]) does not take into account the real needs of the measurement. Or perhaps more precisely, it might; but it is incomplete. An error of $5 \mathrm{mHz}$ rms, spread across the full measurement band as noise, might allow our measurement to be useful, whereas a single interfering signal would masquerade as a system mode. We must ensure that our definitions of the measurand consider the various impairments, both internal to the PMU and in the Smart Grid, that affect our measurement and its applicability.

\section{B. Harmonics vs. broadband noise}

Now consider Fig. 2, which shows a graph of signal frequency vs. amplitude vs. time, in this case the first 40 harmonics of the power system including the fundamental. This is an excellent example of the undesirable effects of pollution on the signal, in this case broadband noise caused by an arc furnace. In the first (lower) part of the plot, the harmonics can be seen, poking above the background noise floor. Then, when the arc furnace starts, the noise floor jumps up and obscures the formerly-visible harmonics.

Figures 3(a), 3(b), and 3(c) show 'slices' of the 3D plot of Fig. 2. The progression from clearly visible harmonics to harmonics almost completely obscured by noise is clear.

What should be done with this? A simple approach (used in most power quality meters) takes the energy in the 'harmonic'

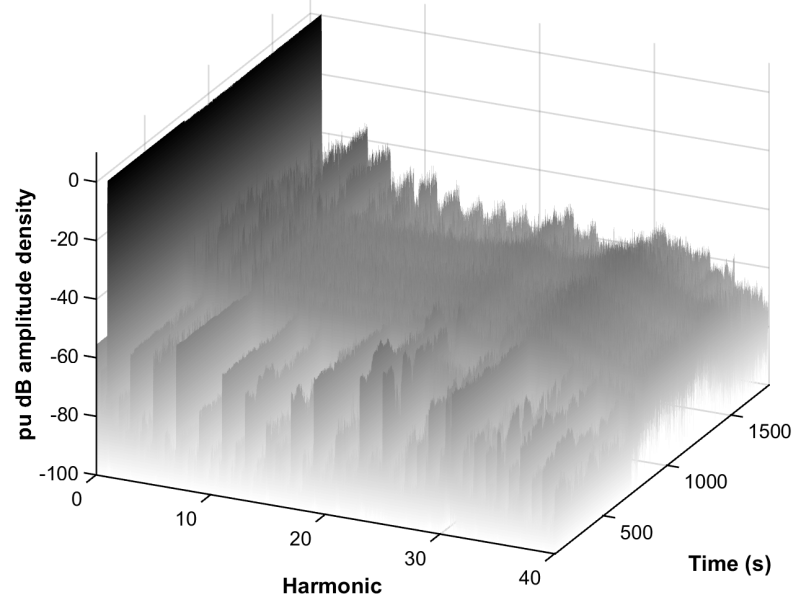

Fig. 2: Harmonic magnitude vs. time, with added noise

bins, and reports that energy as harmonics. Clearly from the more informative view shown by the progression from Fig. 3(a) through 3(c), the harmonics (if they indeed are still there) are no longer readily discernible above the noise floor.

Without an appropriate definition of what to do in a case like this, we might report misleading information to the operator. In this case, the damage is likely to be limited since harmonics (and their accurate measurement) are primarily a customerservice issue. But the issue is broader than simply saying "Only report real harmonics." Some method must be agreed upon regarding how to make the determination of what is real, and what is not.

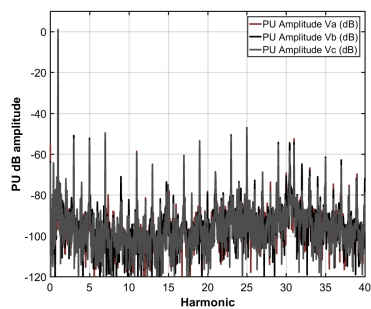

(a) $200 \mathrm{~s}$

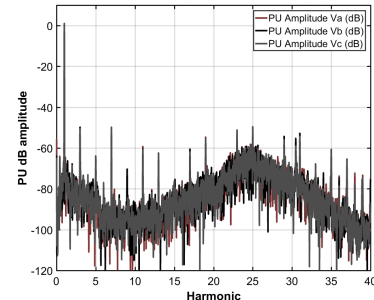

(b) $500 \mathrm{~s}$

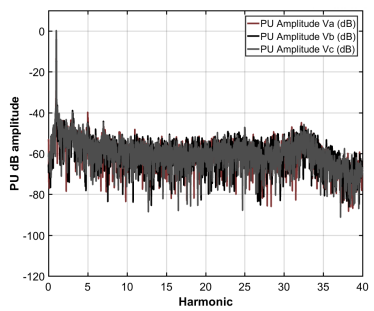

(c) $900 \mathrm{~s}$

Fig. 3: Harmonic magnitude at 3 different times, with noise 


\section{Nuanced data analysis methods}

Our eyes and visual cortex are very powerful tools for data analysis, which is why we use graphs like those shown in the figures above. But when we try to provide a formal definition addressing these issues, we run into problems. Our eyes can put the information in the multiple cascaded plots shown in these figures into a meaningful context, and from that context, we can draw much more nuanced information compared to that which we can infer from a single plot. Even for a single harmonics + noise plot though, the context (noise floor) surrounding the information (harmonic level) provides useful information respecting whether our 'measurement' data are indeed what they appear.

An example of an analytic tool intended to assist with problems like this is the 'peak finder' provided in modern spectrum analyzers, designed to identify peaks surrounded by noise. Indeed, there exist numerous analytical methods to find peaks in data sequences, since this is a problem common to many fields. These tools share the need to set some sort of threshold that enables a 'peak' to be declared. Might this apply to our work and if so, how?

\section{CONCLUSIONS AND FUTURE WORK}

In real physical systems, where we are required to estimate the values of quantities that vary with time, we face a serious problem: we have not, so far, found an agreed-to definition of what we are measuring. Lacking guidance from the GUM or other sources, we must formulate the measurement problem de novo.

We must first agree about what the important characteristics of the quantity are. To do this, we need to fully understand the actual applications of the measurements. Then perhaps we can attempt to formally and unambiguously define the relevant measurands.

We must also consider how to relate the measurands to the ever-present influence quantities. If we add filtering (as we are so far doing), we must understand the resulting effect on the measurement process, as we migrate in an operationalist direction.

This procedure may result in several different definitions for quantities which, until now, have not been well defined. For example, there could be several different formal definitions of frequency, specific to: single-phase/three-phase, required response time (window length), modulation passband width, and the desire to either treat discrete phase-steps as part of the measurand, or as an influence quantity.

We need a succinct and understandable definition of each measurand, that expresses all of these quantities: the basic measurement, the important characteristics of the quantity, and the pollution present. Without this, we will not be able to agree on the overall uncertainty of our results, nor establish their appropriateness for the intended use.

\section{REFERENCES}

[1] "Evaluation of measurement data - Guide to the expression of uncertainty in measurement," JCGM 100:2008. BIPM, Paris, 2008.

[2] Kelvin, William (Lord), "Electrical Units of Measurement" (May 3, 1883), in Popular Lectures and Addresses, pp. 79-136. Macmillan, London, 1889.

[3] "International Vocabulary of Metrology - Basic and General Concepts and Associated Terms," JCGM 200:2012, Joint Committee for Guides in Metrology, WG 1. BIPM, Paris, 2012.

[4] IEC, "Electromagnetic compatibility (EMC) - Part 4-30: Testing and measurement techniques - Power quality measurement methods," IEC 61000-4-30:2015

[5] IEEE, "IEEE Standard for Synchrophasor Measurements for Power Systems," IEEE Std C37.118.1-2011.

[6] H. Kirkham, A. Riepnieks, M. Albu, and D. Laverty, "The Nature of Measurement, and the True Value of a Measured Quantity.” Proc. IEEE IMS I2MTC, 2018.

[7] A. J. Roscoe, S. M. Blair, W. Dickerson, and G. Rietveld, "Dealing with Front-End White Noise on Frequency and ROCOF Measurements in Power Systems," IEEE Transactions on Instrumentation and Measurement, (in 2nd review).

[8] H. Kirkham and A. Riepnieks, "Students' Simple Method for Determining the Parameters of an AC Signal," in 57th Conference on Power and Electrical Engineering (RTUCON). Riga, Latvia, 2016.

[9] H. Kirkham, "Pure and Applied Metrology," IEEE Instrumentation \& Measurement Magazine, pp. 19-24, December 2016.

[10] A. J. Roscoe, G. M. Burt, and G. Rietveld, "Improving frequency and ROCOF accuracy during faults, for P class Phasor Measurement Units," in IEEE Applied Measurements in Power Systems (AMPS), pp. 97-102. Aachen, Germany, 2013.

[11] Wilbraham, Henry, "On a certain periodic function," The Cambridge and Dublin Mathematical Journal, 3: pp. 198-201, 1848.

[12] B. Boashash, "Estimating and Interpreting the Instantaneous Frequency of a Signal-Part 1: Fundamentals," Proc. IEEE, vol. 80, no. 4, pp. 520538, April 1992.

[13] B. Boashash, "Estimating and Interpreting the Instantaneous Frequency of a Signal-Part 2: Algorithms and Applications," Proc. IEEE, vol. 80, no. 4, pp. 540-568, April 1992.

[14] L. Cohen, "Time-Frequency Distributions - a Review," Proc. IEEE, vol. 77, no.7, pp. 941-981, July 1989.

[15] A. J. Roscoe, A. Dyśko, B. Marshall, M. Lee, H. Kirkham, et al., "The Case for Redefinition of Frequency and ROCOF to Account for AC Power System Phase Steps," in IEEE Applied Measurements for Power Systems (AMPS). Liverpool, UK, 2017.

[16] IEEE, "IEEE Standard for Synchrophasor Measurements for Power Systems - Amendment 1: Modification of Selected Performance Requirements," IEEE Std C37.118.1a-2014. 